\title{
Pancreatitis aguda en niños. Una perspectiva desde la autopsia
}

\section{Acute pancreatitis in children. A perspective from the autopsy}

Cecilia Ridaura-Sanz, Arena Arias-Lima

\begin{abstract}
Resumen
OBJETIVO: Determinar la frecuencia, características anatomoclínicas y etiología de la pancreatitis aguda en las autopsias practicadas a los fallecidos en el Instituto Nacional de Pediatría.

MATERIALES Y MÉTODOS: Estudio descriptivo y retrospectivo de serie de casos. Se revisaron todos los expedientes de autopsias del archivo del Departamento de Patología de nuestra institución colectados de 1971 a 2011. Se seleccionaron para estudio los casos de necrosis aséptica del parénquima pancreático y saponificación de la grasa intraglandular y de tejidos vecinos. De los informes postmortem se obtuvieron los datos de la enfermedad principal, las complicaciones y la causa de muerte. De los expedientes clínicos se reunieron los datos demográficos, manifestaciones clínicas, factores de riesgo, diagnósticos clínicos y estudios de laboratorio y gabinete pertinentes. RESULTADOS: De 7250 protocolos de autopsia se identificaron 44 casos con alteraciones histopatológicas de pancreatitis aguda, definida como necrosis aséptica del parénquima pancreático y saponificación de la grasa intraglandular y de tejidos vecinos. La mayoría ocurrió en la última década, lo que sugiere un aumento en la frecuencia de esta enfermedad. Se asociaron a otros padecimientos: 43 de 44 (97\%), predominantemente cáncer y lupus eritematoso sistémico y la etiología más frecuente fue por fármacos. El cuadro clínico característico se describió en el expediente solo en 11 casos (25\%), y el diagnóstico clínico de pancreatitis aguda se documentó en vida solo en 7 casos (16\%). CONCLUSIÓN: La pancreatitis aguda en los niños está subdiagnosticada. Las definiciones y criterios diagnósticos referidos en la bibliografía no toman en cuenta las características de la población con pancreatitis subclínica. Se sugiere la cuantificación de enzimas pancreáticas a todos los pacientes con factores de riesgo para la identificación oportuna de esta complicación.
\end{abstract}

PALABRAS CLAVE: Pancreatitis aguda; autopsia; parénquima pancreático; necrosis aséptica; enfermedad aguda; lupus eritematoso.

\section{Abstract}

OBJECTIVE: To describe the clinical and pathological features acute pancreatitis identified at autopsy, as defined by the presence of aseptic necrosis of pancreatic parenchyma with fat necrosis and saponification of adjacent tissues.

MATERIALS AND METHODS: We identified and reviewed all cases with the pathology diagnosis of acute pancreatitis in our autopsy records of 7250 studies performed from 1971 to 2011.

RESULTS: Forty-four cases were identified, most of them in the last decade, suggesting an increasing frequency of this condition. Only in a single case was pancreatitis the primary disease, in a child dying after a scorpion bite. All other cases were associated with an underlying condition, mostly cancer or systemic lupus erythematosus. The etiology was drug related in most cases. A typical clinical picture was present in only 11 patients (25\%). The clinical diagnosis of acute pancreatitis was established in 7 cases.

CONCLUSION: Acute pancreatitis in children is underdiagnosed. The definitions and clinical criteria as referred in the literature overlook the characteristics of a population with subclinical pancreatitis. Assay of pancreatic enzymes in all patients with risk factors may lead to the timely identification of this condition.

KEYWORDS: Acute pancreatitis; Autopsy; Pancreatic parenchyma; Fat necrosis; Acute disease; Lupus erythematosus.
Departamento de Patología Instituto Nacional de Pediatría, Ciudad de México.

Recibido: 1 de junio 2018

Aceptado: 24 de octubre 2018

Correspondencia

Cecilia Ridaura Sanz cridaura@gmail.com

Este artículo debe citarse como Ridaura-Sanz C, Arias-Lima A. Pancreatitis aguda en niños. Una perspectiva desde la autopsia. Acta Pediatr Mex 2019;40(1):11-5. 


\section{ANTECEDENTES}

La pancreatitis aguda en niños tradicionalmente se ha considerado una enfermedad poco frecuente y de difícil diagnóstico. Las publicaciones recientes insisten en los métodos utilizados para el diagnóstico y en que los criterios para definirla han conseguido mayor precisión para establecer la frecuencia de este padecimiento y sus factores de riesgo..$^{1-3}$ La mayor parte de las casuísticas refiere incremento en la cantidad de casos informados, ya sea por un verdadero aumento en la frecuencia o por mayor reconocimiento clínico. ${ }^{4}$ En las últimas dos décadas en Estados Unidos, México y Australia se reportó un aumento en la incidencia en la población pediátrica. ${ }^{5,6,7} \mathrm{Se}$ estiman 3.6 a 13.2 casos pediátricos por cada millón de individuos-año, incidencia que se asemeja a la de los adultos. ${ }^{4}$

La pancreatitis aguda ocurre en todos los grupos etarios, incluso en lactantes. ${ }^{8,9}$ En México, los informes de pancreatitis aguda en niños son aislados y no hay estudios que informen su prevalencia., ${ }^{710,11}$ Con respecto a la etiología, la pancreatitis en la edad pediátrica difiere de la de los adultos. En estos últimos las causas más comunes son los cálculos biliares y el alcoholismo, pero en los niños se asocia con una amplia variedad de causas reales y potenciales: traumatismos, fármacos, tóxicos-metabólicos, infecciones, enfermedades sistémicas y anomalías congénitas de la región hepatobiliar que dificultan su identificación. ${ }^{7-9}$

Además de la diversidad de agentes causales, no hay una clara definición de su trascendencia clínica, comportamiento y pronóstico. Uno de los problemas para reconocer esta enfermedad en forma integral es su definición. Las hay clínico-patológicas que señalan que la pancreatitis aguda es una inflamación histológica del parénquima pancreático que clínicamente se manifiesta con dolor de aparición abrupta en el abdomen o espalda, acompañado de elevación de las enzimas pancreáticas en la sangre y orina. ${ }^{1,2}$ Otra definición propuesta por el INSPPIRE (International Study Group of Pediatric Pancreatitis: in Search for a Cure) señala como criterio diagnóstico dos de tres de los siguientes datos: 1 ) Dolor abdominal agudo, sobre todo en la región epigástrica; 2) concentraciones de amilasa o lipasa tres veces por arriba del valor normal y 3 ) hallazgos por imagen de alteraciones pancreáticas características o sugerentes de pancreatitis aguda. ${ }^{3}$ Estos y otros criterios, que son los que se aplican para describir las características de este padecimiento, solo permiten incluir a un segmento de la población afectada, que es el de los casos documentados clínicamente.

Quizá la descripción más concreta y útil que incluye la fisiopatología del daño es la señalada por García y su grupo, en la que definen a la pancreatitis aguda como una "enfermedad inflamatoria del páncreas exocrino, causada por la activación, liberación intersticial y autodigestión de la glándula por sus propias enzimas".$^{12}$ La expresión histopatológica es la de necrosis aséptica del parénquima pancreático y la saponificación de la grasa intraglandular y de los tejidos vecinos. Con esta definición objetiva es posible identificar los casos en estudios postmortem, independientemente del cuadro clínico y de laboratorio.

\section{OBJETIVO}

El objetivo de este estudio fue: determinar la frecuencia, características anatomoclínicas y etiología de la pancreatitis aguda en las autopsias practicadas a población pediátrica atendida en el Instituto Nacional de Pediatría.

\section{MATERIALES Y MÉTODOS}

Estudio descriptivo y retrospectivo de serie de casos. Se revisaron todos los expedientes de autopsias del archivo del Departamento de 
Patología del Instituto Nacional de Pediatría colectados de 1971 a 2011. Se seleccionaron todos los casos con diagnóstico de pancreatitis consignado en los registros anatómicos finales. Para corroborar el diagnóstico de pancreatitis aguda por necrosis aséptica del parénquima pancreático y la saponificación de la grasa intraglandular y de los tejidos vecinos, se revisaron las fotografías macroscópicas y preparaciones histológicas del páncreas. Los datos de la enfermedad principal, las complicaciones y la causa de muerte se obtuvieron de los reportes postmortem. De los expedientes clínicos se obtuvieron los datos demográficos, manifestaciones clínicas, factores de riesgo, diagnósticos clínicos y estudios de laboratorio y gabinete pertinentes.

\section{RESULTADOS}

De la revisión de 7250 protocolos de autopsia se encontraron 44 casos que cumplieron los criterios histológicos de pancreatitis aguda con necrosis del parénquima y de la grasa adyacente y que correspondieron a $0.6 \%$ de las autopsias colectadas en 40 años. La frecuencia relativa de los casos en relación con la cantidad de autopsias por décadas se expresa en el Cuadro 1, donde se advierte un incremento considerable de casos en la última década. La mayor parte se encontró en escolares y adolescentes, con edad promedio de 9.9 años (desviación estándar 4.4), mediana de 11 años (rango intercuartilar: lar (p25-p75): 8-11). Predominó el sexo femenino 29 vs 15. Las enfermedades de base se expresan en el Cuadro 2. Solo en la muerte por picadura

Cuadro 1. Frecuencia de pancreatitis aguda

\begin{tabular}{|l|c|c|c|}
\hline & \multicolumn{3}{c|}{ Distribución por décadas } \\
\hline Decenio & $\mathbf{n}$ & Autopsias & $\%$ \\
\hline $1970-1979$ & 13 & 2936 & 0.4 \\
\hline $1980-1989$ & 6 & 2591 & 0.2 \\
\hline $1990-1999$ & 6 & 1081 & 0.6 \\
\hline $2000-2010$ & 16 & 639 & 2.5 \\
\hline & 41 & 7238 & 0.5 \\
\hline
\end{tabular}

Cuadro 2. Enfermedad de base

\begin{tabular}{|l|c|c|}
\hline Padecimiento & n & \% \\
\hline Cáncer & 15 & 34.0 \\
\hline Lupus eritematoso & 8 & 18.1 \\
\hline Infección* & 6 & 13.6 \\
\hline Inmunológico & 4 & 9.0 \\
\hline Fiebre reumática & 3 & 6.8 \\
\hline Glomerulonefritis & 2 & 4.5 \\
\hline Otros** & 6 & 13.6 \\
\hline Total & $\mathbf{4 4}$ & $\mathbf{9 9 . 6}$ \\
\hline
\end{tabular}

*Hepatitis, ${ }^{2}$ leptospirosis, ${ }^{1}$ tuberculosis, ${ }^{1}$ sarampión, ${ }^{1}$ sida ${ }^{* *}$ glucogenosis tipo I, ${ }^{1}$ epilepsia, ${ }^{1}$ lipofuchinosis, ${ }^{1}$ esferocitosis hereditaria, ${ }^{1}$ neumonía por aspiración, ${ }^{1}$ picadura de alacrán ${ }^{1}$

de alacrán, la pancreatitis fue la enfermedad principal, en el resto se encontró asociada con otro padecimiento. El factor de riesgo más frecuente en los casos de pancreatitis y posible etiología fue la administración de fármacos, sobre todo esteroides antineoplásicos y anticonvulsivantes (Cuadro 3). Otro caso que merece la pena señalar es el que ocurrió en un niño con glucogenosis tipo I (enfermedad de VonGierke) con hiperlipidemia elevada, que fue lo que le ocasionó la muerte por la lesión pancreática. En nueve casos no se encontró la causa y se consideraron idiopáticos.

Cuadro 3. Etiología

Fármacos

$33(75 \%)$

Esteroides

21

Esteroides y asparaginasa

Esteroides y aziatropina 2

Esteroides y azatioprina más ácido valproico $\quad 1$

Ácido valproico

Antineoplásicos

Toxina $1(2.2 \%)$

Veneno de alacrán

Metabólica

$1(2.2 \%)$

Hiperlipidemia

Se ignora $9(20 \%)$ 
Por lo que se refiere al cuadro clínico, en 16 de 44 casos no se describen en los expedientes manifestaciones clínicas abdominales sugerentes de pancreatitis (dolor abdominal con o sin vómito). La mayoría de estos casos se encontraba en estado crítico por su enfermedad de base y permanecían sedados o intubados. De los casos sintomáticos, 11 tuvieron el cuadro clínico característico que se refiere en la bibliografía (Cuadro 4). El diagnóstico en vida de pancreatitis se estableció en siete casos. Todos tuvieron el cuadro clínico y en cinco se documentó la elevación de la amilasa y lipasa. Un caso tuvo cifras normales de amilasa pero no se efectuó la determinación de lipasa.

Cuadro 4. Cuadro clínico

\begin{tabular}{|l|c|c|}
\hline Síntomas & $\mathbf{n}$ & $\%$ \\
\hline Asintomático & 16 & 36.3 \\
\hline No relacionado & 17 & 38.6 \\
\hline Cuadro típico & 11 & 25.0 \\
\hline Total & $\mathbf{4 4}$ & $\mathbf{9 9 . 9}$ \\
\hline
\end{tabular}

\section{DISCUSIÓN}

Si bien la población con autopsia de este hospital no es representativa de la totalidad de la mortalidad y seguramente concentra los casos más graves, algunos de los resultados sí pueden extrapolarse porque no parece probable que se hayan seleccionado los casos por la patología pancreática.

En los últimos años se ha incrementado la frecuencia de la pancreatitis aguda porque han aumentado los casos de enfermedades que conducen a esta complicación, sobre todo por el consumo de fármacos reconocidamente tóxicos para el páncreas, como los esteroides y antineoplásicos. ${ }^{13} \mathrm{~A}$ pesar de tratarse de una población pediátrica no encontramos casos se- cundarios a malformaciones del árbol biliar, ni lesiones por traumatismo, que son las referidas como frecuentes en esta población. De los 44 casos estudiados solo en uno fue el padecimiento primario y causante de la muerte por picadura de alacrán.

Esta complicación se ha informado en modelos experimentales y clínicos. ${ }^{14}$ En el resto de los casos la pancreatitis ocurrió concomitante con enfermedades graves sistémicas y muchas se trataron con fármacos potencialmente tóxicos. En nueve casos no se encontró la causa directa de la pancreatitis y se consideró idiopática. Conforme se conocen nuevos mecanismos de la enfermedad, como los genéticos de los individuos y de las poblaciones, esta clasificación ha ido desapareciendo. En este sentido se ha demostrado la diversa susceptibilidad para padecer pancreatitis y se encontró que es más frecuente en ciertos grupos étnicos, como los del Reino Unido en donde se registra mayor frecuencia en el grupo poblacional pakistaní. ${ }^{14}$ También se han descrito algunos genes asociados con mayor susceptibilidad al daño pancreático. ${ }^{15}$

Uno de los aspectos sobresalientes de este estudio es el subdiagnóstico de la pancreatitis aguda porque solo 16\% se identificó en pacientes vivos. Esta falla diagnóstica puede explicarse por la falta de síntomas abdominales en el curso de la enfermedad de base, ya sea porque no existieron o porque los oscurecieron los síntomas subyacentes. En 11 casos se refirió un cuadro clínico típico que ante un factor de riesgo debió haber alertado para confirmar el daño pancreático. Se propone que ante factores de riesgo con los medicamentos reconocidamente tóxicos pancreáticos se realice la determinación de la lipasa y amilasa para establecer el diagnóstico de pancreatitis. La hiperlipidemia es otro factor de riesgo relevante, como sucedió en el niño con glucogenosis tipo I, en niños mexicanos obesos en quienes debe llevarse un control cercano de la función pancreática para prevenir complicaciones. ${ }^{16}$ 
De los casos de infección sobresalen los asociados con hepatitis fulminante en las que no se pudo establecer la causa del daño hepático. ${ }^{17}$

\section{CONCLUSIONES}

En la última década aumentó la frecuencia relativa de pancreatitis aguda en niños, quizá por incremento en la frecuencia de padecimientos que requieren tratarse con fármacos potencialmente tóxicos para el páncreas. Ante el subdiagnóstico clínico puesto de manifiesto en este estudio es importante reconocer esta complicación en casos con factores de riesgo, independientemente del cuadro clínico. En pacientes en tratamientos prolongados con esteroides o antineoplásicos es pertinente la determinación periódica de las enzimas pancreáticas con el propósito de detectar esta enfermedad en la población pediátrica e iniciar el tratamiento oportuno. Los estudios de autopsia proporcionan información más amplia acerca de esta enfermedad.

\section{REFERENCIAS}

1. Banks PA, Bollen TL, Dervenis C, et al. Classification of acute pancreatitis 2012: Revision of the Atlanta classification and definitions by international consensus. Gut. 2013;62(1):102-11.

2. Koizumi M, Takada T, Kawarada Y, et al. Guidelines for the management of acute pancreatitis: diagnostic criteria for acute pancreatitis. J Hepatobiliary Pancreat Surg. 2006;13(1):33-41.

3. Morinville VD, Husain SZ, Bai H, et al. Definitions of pediatric pancreatitis and survey of current clinical practices: Report from INSPPIRE (International Study Group OF Pediatric Pancreatitis: IN Search FOR A Cure). Pediatr Gastroenterol Nutr. 2012;55(3): 261-65.
4. Majbar AA, Cusck E, Johnson P, et al. The incidence and clinical associations of childhood acute pancreatitis. Pediatrics. 2016;138 (3):e20161198.

5. Morinville VD, Barmada MM, Lowe ME. Increasing incidence of acute pancreatitis at an American pediatric tertiary care center: is greater awareness among physicians responsible? Pancreas. 2010;39(1):5-8.

6. Nydegger A, Heine RG, Ranuh R, et al. Changing incidence of acute pancreatitis: 10-year experience at the Royal Children's Hospital, Melbourne. J Gastroenterol Hepatol. 2007;22(8):1313-16.

7. Sánchez-Ramírez CA, Larrosa-Haro A, Flores-Martínez S, et al. Acute and recurrent pancreatitis in children: etiological factors. Acta Paediatr. 2007;96(4):534-37.

8. Srinath Al, Lowe ME. Pediatric Pancreatitis. Pediatr Rev. 2013;34(2):79-90.

9. Mekitarian E, Carvalho W, Duarte F. Acute pancreatitis in pediatrics: a systematic review of the literature. J Pediatr (Rio J). 2012;88(2):101-14.

10. Sánchez-Lozada R, Camacho-Hernández MI, VegaChavaje RG. Pancreatitis aguda: experiencia de cinco años en el Hospital General de México. Gac Med Mex. 2005;141(2):123-27.

11. Sánchez AC, García Aranda JA. Pancreatitis aguda. Bol Med Hosp Infant Mex. 2012;69(1):3-10.

12. García Burriel Jl, Vilar Escrigas PJ. Pancreatitis en el niño. Protocolos diagnóstico-terapéuticos de Gastroenterología, Hepatología y Nutrición Pediátrica. SEGHNP-AEP. Ergon, 2010;135.

13. Stefanovic $\mathrm{M}$, Jazbec J, Lindgren $\mathrm{F}$, et al. Acute pancreatitis as a complication of childhood cancer treatment. Cancer Medicine 2016;5(6):827-36.

14. Venancio EJ, Portaro FCV, Kuniyoshi AK, et al. Enzymatic properties of venoms from Brazilian scorpions of Tityus genus and the neutralisation potential of therapeutically antivenoms. Cancer Medicine 2016; 5 (2):180-90.

15. Whitcomb DC. Genetic risk factors for pancreatic disorders. Gastroenterology. 2013;144(6):1292-302.

16. Yadav D, Pitchumoni CS. Issues in hyperlipidemic pancreatitis. J Clin Gastroenterol. 2003;36(1):54-62.

17. Srinivasan A, Venkataraman S, Hansdak SG, et al. Hyperglycaemia as an indicator of concurrent acute pancreatitis in fulminant hepatic failure associated with hepatitis B infection. Singapore Med J. 2005; 46(5):236-37. 